\title{
Institutionalisierung und Transformation: Bildungs- und Lernprozesse in und von (Protest-)Bewegungen
}

\begin{abstract}
Zusammenfassung
Wie die derzeitige Corona-Pandemie verdeutlicht, zeichnen sich Krisenzeiten durch ihren spezifischen Charakter und die damit verbundene gesellschaftliche und politische Bearbeitung, durch eine besondere Dynamik, aus. Oft tritt auch verstärktes oder stärker sichtbares zivilgesellschaftliches Engagement auf, welches wiederum mit weiteren gesellschaftlichen Veränderungen einhergehen kann, wie beispielsweise Proteste oder Demonstrationen und die damit ausgelösten Anstöße zu politischen oder medialen Debatten. Die Akteur/-innen, wie derzeit zum Beispiel die Fridays-for-Future-Bewegung, sind für gezielten Wandel wichtig, aber auch organisationale Strukturen und der Kontext (Gelegenheitsstrukturen), die zivilgesellschaftliches Handeln (mit-)bestimmen. Welche Bedeutung haben soziale Bewegungen für eine nachhaltige gesellschaftliche Entwicklung und inwiefern können sie als gesellschaftliche und individuelle Lern- und Bildungsräume verstanden werden?

In diesem Beitrag wird, in Anschluss an die Darstellung zentraler Theoriestränge sozialwissenschaftlicher Bewegungsforschung, illustriert, wie gesellschaftliche Transformation und Engagement auch als aufeinander bezogene Lern- und Bildungsprozesse in und von Bewegungen verstanden werden können. Dies wird anhand der Ökologiebewegung und der Fridays-for-Future-Bewegung aufgezeigt.
\end{abstract}

Schlüsselworte: Klimakrise, Fridays for Future, Bildungsprozesse, Lernräume, soziale Bewegungen

\footnotetext{
Abstract

The corona-pandemic demonstrates that times of crisis are characterized by their specific dynamic especially through their social and political processing. They are often accompanied by an increased or more visible civil society engagement. This can result in further social changes, such as protests or demonstrations resulting in political or media debates. The actors involved - e. g. the current Fridays for Future movement - are important for the targeted change, as well as the organizational structures and the context (opportunity structures) that determines civil society actions.
}

\begin{abstract}
What significance do social movements have for sustainable (social) development and to what extent can they be understood as social and individual learning or educational spaces?

In this paper, I present central theoretical strands of the research on social movements and their development. Then I focus on the associated learning and educational processes in and for movements and the actors involved. The example of ecological movements and Fridays for Future illustrates how social transformation and individual engagement and learning are related to one another.
\end{abstract}

Keywords: Climate Crisis, Education, Fridays for Future, Social Movements

\section{Einleitung}

Gerade Krisenzeiten zeigen durch ihren spezifischen Charakter und der damit verbundenen erforderlichen gesellschaftlichen und politischen Bearbeitung eine besondere Dynamik. Die Corona-Pandemie und deren politische und gesellschaftliche Be- und Verarbeitung sind ein aktuelles Beispiel dafür, wie sich zivilgesellschaftlicher und politischer Aktivismus gegenwärtig konstituiert oder verändert: So rief die noch junge Bewegung der Fridays for Future (FFF) im Jahre 2019 zu wöchentlichen Schulstreiks und großen Demonstrationen (Klimastreiks) auf. Aufgrund der politischen Maßnahmen zur Pandemieeindämmung verlagerten die Aktivist/-innen ihre Streiks ins Internet, organisierten Online-Streiks oder Protest-Camps und versuchten mit coronakonformen Mitteln ihr Engagement zur Begegnung der Klimakrise zu organisieren. Andere Personen oder Gruppierungen, wie z.B. Coronaleugnende oder Maßnahmenkritisierende, u.a. die so genannten Querdenker, brachen dagegen mit den von der Politik verhängten Hygieneregeln oder organisierten sich gegen die Maßnahmen (wie Testungen oder Impfungen). ${ }^{1}$ Auch wenn diese in diesem Beitrag nicht näher beleuchtet werden, so verweist die Gegenüberstellung auf eine veränderte oder verstärkte Dynamik im Engagement sowie in den Mitteln und Formen von Protest. Ebenso zeigen gegenüberstehende oder kontroverse Aktivismen eine gesellschaftliche Dynamik auf, welche wiederum weitere ge- 
sellschaftliche oder politische Veränderungen einläuten könnte. In diesem Beitrag werden zuerst zentrale Theoriestränge sozialwissenschaftlicher Bewegungsforschung und informeller Lern- und Bildungsprozesse dargestellt. Die Fallbeispiele der frühen Ökologiebewegungen und FFF bieten die Möglichkeit zu zeigen, wie Bewegungen sich transformieren und zugleich einen Ort und Rahmen bieten können, in dem junge Menschen politisch aktiv werden und dadurch selbst Lern-und Bildungsräume durch Aktivismus und Engagement eröffnen. Sie illustrieren, wie gesellschaftliche Transformation und zivilgesellschaftliches Engagement auch als aufeinander bezogene, interdependente Lern- und Bildungsprozesse verstanden werden können.

\section{Sozialwissenschaftliche Bewegungsfor- schung}

Soziale Bewegungen sind seit den 1960er-Jahren - mit den so genannten Neuen sozialen Bewegungen - verstärkt Gegenstand sozialwissenschaftlicher Forschung geworden. Darüber, was genau eine soziale Bewegung im Detail charakterisiert, besteht keine Einigkeit (Ahlemeyer, 1989; Amenta, 2002). Dabei ist die Untersuchung des Phänomens stark an ihren historischen Kontext und an gesellschaftliche Kernfragen gebunden. In zahlreichen wissenschaftlichen Auseinandersetzungen werden soziale Bewegungen recht unterschiedlich definiert, weshalb fraglich erscheint, ob jeweils von dem gleichen Phänomen ausgegangen wird, oder aber, ob der Begriff der sozialen Bewegung vielmehr zu einem Sammelbegriff geworden ist, der vielfältige Formen von kollektivem Aktivismus, oder recht unterschiedliche Formen von Zusammenschlüssen von Akteur/-innen, gleichermaßen beinhalten kann. Die jeweiligen Definitionen bilden den Ausgangspunkt bzw. orientieren die empirischen und theoretischen Auseinandersetzungen zu unterschiedlichen Konzeptualisierungen des untersuchten Phänomens. Trotz der Unterschiede in den Definitionen, lässt sich als Kern der meisten Ansätze ein kollektiver Akteur ausmachen, der um gesellschaftlichen Wandel bemüht ist. Ziele, Organisationsgrad, die Dauerhaftigkeit und weitere Eigenschaften sind hingegen in den verschiedenen Definitionen unterschiedlich gefasst. Nach Rucht gelten soziale Bewegungen „als ein auf gewisse Dauer gestellter Versuch mobilisierter Netzwerke von Gruppen und Organisationen, sozialen Wandel durch Protest herbeizuführen, zu verhindern oder rückgängig zu machen “ (1994, S. 22f.). Von der Autorengruppe Rucht et al. (1998) wird dezidiert auf den Unterschied zwischen der Forschung zu sozialen Bewegungen und der Protestforschung verwiesen und sie merken an, dass sich soziale Bewegungen zwar oftmals des Protests als Mittel zur Durchsetzung sozialen Wandels bedienen, dass aber Protest nicht unbedingt mit dem Auftreten sozialer Bewegungen verbunden sein muss.

Eine empirische Frage ist, wie das Verhältnis von Protest und sozialer Bewegungen charakterisiert ist, aber auch, wie und was Bewegungen verhilft, erfolgreich sozialen Wandel anzustoßen. Dazu hat die sozialwissenschaftliche Bewegungsforschung theoretische Ansätze entwickelt, die sich auf soziale und politische Akteur/-innen, soziale Bewegungen und ihre Wirkungen, Entwicklungen und Erfolge sehr unterschiedlich und anhand diverser Fragestellungen beziehen. McAdam et al.
(1996) verweisen darauf, dass oftmals nur ausgewählte Aspekte in den Untersuchungen Berücksichtigung fanden. Jeder Ansatz wird im Grunde dafür kritisiert, andere Aspekte zu vernachlässigen oder auszublenden. Eine Herausforderung scheint daher darin zu liegen, die theoretischen Konzepte aufzunehmen, zu kombinieren und zu erweitern und ein umfassendes Verständnis sozialer Bewegungen und ihrer eigenen sowie der gesellschaftlichen oder politischen Dynamik zu erlangen. Im Kontext des Beitrags geht es darum, soziale Bewegungen als Orte von und für Bildung und Lernen zu begreifen, die erst durch die Dynamik und Prozesshaftigkeit als solche in Erscheinung treten.

\section{Transformationsprozesse sozialer Bewegungen}

Soziale Bewegungen zeichnet ihre Dynamik und Prozesshaftigkeit aus. Aus sozialen Bewegungen oder aus Teilen derselben können z.B. zivilgesellschaftliche oder politische Akteur/-innen hervorgehen (wie Interessengruppen oder politische Parteien). Ein Beispiel, das den Prozess der Institutionalisierung von Teilen einer Bewegung hin gut illustriert, sind die Grünen Parteien, die u.a. aus Umwelt- und Anti-Atomkraftbewegungen oder wie in Österreich aus Bürger/-inneninitiativen hervorgegangen sind.

In der Bundesrepublik Deutschland entwickelte sich aus dem Bewegungsmilieu die Partei Bündnis 90/Die Grünen, welche sich schließlich im Parteiensystem etablierte (Roth, 1991, S.435). Offe beschreibt diesen Prozess der Institutionalisierung der deutschen Grünen: „Socio-political movements as forms of collective action usually start in an institutional vacuum, with no other institutional resources available to them then the usually partly contested legal and constitutional rights of citizens to assemble, communicate, protest, petition, and demonstrate" (1990, S. 236).

Die Entwicklungs- und Transformationsprozesse sozialer Bewegungen werfen Fragen nach dem Verhältnis der Akteur/-innen und ihrem Kontext, aber insbesondere auch anderen Akteur/-innen auf (wie politischen Parteien oder auch politischen Eliten). Parteien und Bewegungen werden von Rucht als „unterscheidbare, aber gleichwohl aufeinander bezogene Typen kollektiven Handelns" (1987, S. 297) beschrieben. $\mathrm{Ab}$ welchem Grad von Institutionalisierung oder anhand welcher Aspekte soziale Bewegungen nicht mehr als solche bezeichnet werden können, ist keineswegs feststehend.

Das Spektrum reicht von losen oder spontanen Aktionen über strukturiertere Zusammenschlüsse bis hin zu etablierten Social Movement Organizations, sog. Bewegungsorganisationen, die von McCarthy und Zald als „complex, or formal, organization which identifies its goals with the preferences of a social movement or countermovement and attempts to implement those goals" (1977, S. 153) beschrieben wurden. Transformationsprozesse sozialer Bewegungen werden einerseits vom Kontext, andererseits von der Entwicklung der Bewegung und ihrer Akteur/-innen selbst bestimmt.

Die Veränderungen einer sozialen Bewegung können zwar bedeuten, dass sich die Bewegung institutionalisiert und sich zum Beispiel als politischer oder zivilgesellschaftlicher Akteur etabliert (wie eine Partei oder Nichtregierungsorganisation, 
NGO), sie kann aber auch deren Zerfall oder Zersplitterung bedeuten, wobei davon Teile oder die gesamte soziale Bewegung betroffen sein können. Diese sei an drei Entwicklungstendenzen erkennbar: am Übergang zu Sekten oder Subkulturen, der Institutionalisierung in Parteien oder Verbänden oder am Übergang in andere soziale Bewegungen (Ebbinghaus, 2008, S.16f.). Das Ende einer sozialen Bewegung bzw. die Transformation hin zu einem anderen zivilgesellschaftlichen oder politischen Akteur hängt damit zusammen, wie soziale Bewegungen als Phänomen definiert werden und welche Faktoren dafür als entscheidend angesehen werden. Es ist dabei aber nicht zuletzt eine empirische Frage, deren Beantwortung in der Analyse von Bewegungen, ihrer Interaktion mit anderen (politischen, zivilgesellschaftlichen) Akteur/-innen und in konkreten gesellschaftlichen Prozessen bzw. Kontexten (Gelegenheitsstrukturen) liegt. Auch die Erhebung empirischer Daten $\mathrm{zu}$ Bewegungen steht vor besonderen Herausforderungen, denen sie mit spezifischen Strategien, wie z.B. durch die Befragung von Protestteilnehmenden oder Aktivist/-innen, begegnet. Zusammenfassend sind soziale Bewegungen dynamische Phänomene, die sich nicht nur in ihrer Organisationsstruktur verändern können. Bewegungen als prozesshafte und fluide Phänomene stellen die sozialwissenschaftliche Erforschung vor besondere Herausforderungen und definitorische Fragen.

Die Prozesshaftigkeit von Bewegungen selbst, das heißt, die Entwicklung und potenzielle Transformation oder Institutionalisierung, liegt in einem interdependenten Verhältnis zwischen Bewegung und Akteur/-innen, sowie dem Kontext und den politischen Gelegenheitsstrukturen begründet. Dazu können beispielsweise Allianzen und Verbindungen zu anderen zivilgesellschaftlichen Akteur/-innen zählen. Dadurch eröffnen soziale Bewegungen Räume für potentielle Professionalisierung und Institutionalisierung begleitet von informellen Lern- und Bildungsprozessen der Akteur/-innen.

\section{Soziale Bewegungen als Lern- und Bildungsräume}

Verschiedene Faktoren wirken auf die Entwicklung einer Bewegung oder Bewegungsorganisation: z.B. die Ziele der Mitglieder, die Erwartungen und Aussichten innerhalb und außerhalb der Bewegung. Die Akteur/-innen haben neben kollektiven auch subjektive Zugänge, Ambitionen oder individuelle Ziele. Diese sind wiederum innerhalb einer größeren Struktur zu verorten, die von multiplen (wie strukturellen oder individuellen) Faktoren abhängen. Um die Interdependenzen zwischen den verschiedenen Ebenen, der Akteur/-innen, der Organisationsstruktur und ihres gesellschaftlichen und politischen Kontextes sichtbar zu machen, werden diese im Folgenden nicht nur theoretisch, sondern auch anhand konkreter, empirischer Fälle dargestellt. ${ }^{2}$

Am Beispiel von Umweltaktivismus kann verdeutlicht werden, wie sich einerseits Institutionalisierungsprozessen sozialer Bewegungen vollzogen haben und in der Gründung und Konstituierung von Parteien und NGOs resultierte. Andererseits zeigt es, wie Gelegenheitsstrukturen und aktuelles Engagement durch Aktivist/-innen wie jene der FFF (in anderer Weise z.B. auch Ende Gelände oder System Change, not Climate Change) mit individuellen und kollektiven Transformationsund Bildungsprozessen zusammenhängen.
Obwohl zwischen Bildung und sozialen Bewegungen starke Verbindungen bestehen, wird dieser Zusammenhang bislang eher selten untersucht (Miethe \& Roth, 2016, S. 20). Dies liegt auch an den teils unverbundenen wissenschaftlichen Diskursen zu sozialen Bewegungen seitens der Soziologie, Politikwissenschaft oder Sozialpsychologie auf der einen und erziehungs- oder bildungswissenschaftlichen Diskursen zu informellem Lernen und Bildungsprozessen auf der anderen Seite. In der Forschung zu sozialen Bewegungen werden Bildungsprozesse nur selten explizit erwähnt und der dort zugrunde gelegte Bildungsbegriff erschließt sich allenfalls implizit (Miethe \& Roth, 2016, S. 20). In der Erziehungswissenschaft wird zwischen den Begriffen des Lernens und der Bildung unterschieden und unter Rückgriff z.B. auf einen transformatorischen Bildungsbegriff (Koller) argumentiert. Miethe und Roth (2016, S. 21) verstehen Lernen beispielsweise eher als Ansammlung von Wissen oder von Kompetenzen, während der Bildungsbegriff eher als Prozess gerahmt wird, wie Wissen eine neue oder eigenständige Qualität erhält. Der Begriff der Bildung nimmt den Menschen ganzheitlich und nicht ausschließlich als rationale/n Akteur/-in in den Blick. Er legt den Schwerpunkt auf den Prozess der eigenen (inneren) Weiterentwicklung und Selbstdeutung. Bildungsprozesse umfassen so notwendigerweise Lernprozesse, da die Lernfähigkeit die Grundlage für Bildungsprozesse darstellt.

Lernen und Bildung bedingen sich somit. Zudem können sie sowohl individuelle als auch kollektive Prozesse beinhalten. Zwei Richtungen transformativen Lernens lassen sich nach Singer-Brodowski (2016, S. 15) unterscheiden, wobei beide Richtungen Ansätze der kritischen Theorie und der sozial-konstruktivistischen Lerntheorien integrieren: die Ansätze transformativen Lernens als Wandel individueller Bedeutungsperspektiven und die Konzepte des transformativen Lernens als kollektiver Bewusstwerdungs- und Emanzipationsprozess.

Die Rolle informeller Lernprozesse, als Lernen in konkreten Lebenszusammenhängen (z.B. in Arbeitszusammenhängen oder im politischen oder sozialen Engagement selbst) rückt dabei besonders in den Fokus und es schließen sich Diskurse um deren Funktion und Bedeutung an (Overwien, 2013). Informelles Lernen erscheine nach Overwien (2016, S. 400) nicht als qualitativ besseres Lernen und könne durchaus auch fehlerhaft sein. Es trage oft autodidaktische Züge und die informellen Lernprozesse in sozialen Bewegungen erfolgen zumeist unbewusst, bzw. werden von den Aktivist/-innen nicht unbedingt als Lernen wahrgenommen. Dies, obwohl sie sich durch ihr Engagement etwa Fachwissen zu dem jeweiligen politischen Feld, in dem die Bewegung aktiv ist, aneignen oder soziale Kompetenzen ebenso wie Analyse- oder Medienkompetenzen erwerben. Dadurch entstünde ein erhöhtes Bedürfnis nach organisierten, formellen Lernprozessen (Overwien 2013, S. 6ff.). Es ist aber in seiner Bedeutung für die Subjekte ebenso wie in seiner anregenden und gemeinschaftsstiftenden Funktion von zentraler Bedeutung. Für Protestbewegungen beschreibt Haselwanter (2020, S. 14), dass sich temporäre Fenster für Möglichkeiten des Erkenntnisgewinns und der dezidierten inhaltlichen Auseinandersetzung ergäben. Ein Charakter von Bildung rücke in den Mittelpunkt, für den weder Vorkenntnisse noch das Alter wesentlich erscheinen, sondern ein integrativer Umgang zwischen allen Beteiligten. Da- 
mit treten Möglichkeiten für Bildungsprozesse und -räume in der und durch die Bewegung auf, die Beziehungen zwischen Akteur/-innen unterschiedlicher Generationen innerhalb von Bewegungen zulassen und (Weiter-)Bildungsprozesse auslösen. Soziale Bewegungen, so Miethe (2017), könnten folglich eine Selbstermächtigung bedeuten und identitätsbildend wirken. Durch die Aktivität in sozialen Bewegungen würden Personen direkt Kompetenzen erwerben, die sie z.B. im Studium bzw. auf dem Weg dorthin nutzen könnten: Um die Bewegung aufrecht zu erhalten, müssen die Mitglieder und Sympathisant/-innen die Selbstorganisation vorantreiben und z.B. Veranstaltungen und Öffentlichkeitsarbeit organisieren, sich in Diskussionen einbringen und argumentieren, was mit Kompetenzerwerb einhergehe (Miethe, 2017, S. 36).

Overwien (2013) unterstreicht, ebenso wie Singer-Brodowski (2016), Überschneidungen mit der Educación Popular im Sinne Paulo Freires. Mit Blick auf die Interessen lernender Subjekte könne an diese Denktradition angeschlossen werden, insofern die eigene Betroffenheit wie z.B. durch ein Atomkraftwerk oder die Durchführung von Infrastrukturprojekten zur Erkennung generativer Themen führe und von dort weiter zu Lernprozessen über Zusammenhänge, zu Kommunikation über die Aktion als kritische Praxis, zur Aneignung von Politikkompetenz, die gemeinsam ohne übliche Lernhierarchien erarbeitet wird. Praxis und Theorie würden so ineinander übergehen, wobei die je für die Argumentationen und für das politische Handeln notwendigen Theorien sich immer wieder angeeignet werden müssen (Freire, 1973; Overwien, 2013, S. 404). Eine Untersuchung Rodemanns (2009) z.B. zeigt, wie sich informelles Lernen gestaltet: Greenpeace-Aktivist/-innen, welche zum Zeitpunkt der Befragung jeweils einige Jahre lang in entsprechende Prozesse integriert waren, gaben an, dass insbesondere kommunikative und auf Umweltfragen bezogene Kompetenzen erworben wurden. Die Studie von Truemann (2013) zeigt, wie Bürger/-inneninitiativen dem/der Einzelnen die Chance zu politischem Lernen und Handeln bieten, das Feld zu politischen Partizipationsformen öffnen, aber auch Alternativen zum Bisherigen zu denken und die Wirksamkeit eigenen Handelns zu erleben. Die Autorin verweist dabei auf utopisches Denken und darauf, über die Kritik am Bestehenden auch zur Entwicklung von Gegenentwürfen zu kommen. Dies weist große Übereinstimmungen mit den Zielen einer Bildung für nachhaltige Entwicklung oder Globalen Lernens auf.

\section{Umwelt und Fridays for Future}

Die Geschichte des umweltpolitischen Engagements illustriert Institutionalisierungs- und Professionalisierungsprozesse und zeigt die individuellen und strukturellen Besonderheiten der FFF in Österreich als Fortführung und Abgrenzung dazu. ${ }^{3}$ So zählen Klima und Umwelt laut Dolezal (2019) in Österreich zu jenen Themen, die eine besondere Mobilisierungswirkung aufweisen. Ab den 1960er-Jahren kam es u.a. zu Auseinandersetzungen mit postmateriellen Werten und auch das ökologische Bewusstsein für Gefahren und die Begrenztheit der natürlichen Ressourcen nahm zu (Pelinka, 1991; Gottweis, 1997). Das politische System und die Dominanz von SPÖ und ÖVP wurden herausgefordert, da es den Parteien stetig weniger gelang, Wähler/-innen zu erreichen (Pelinka, 1991). Den etablierten politischen Parteien standen Aktivist/-innen gegenüber, die sich für mehr Bürger/-innenbeteiligung und die Verankerung von Umweltschutz in der Politik einsetzten (Gottweis, 2000). Zwei Ereignisse und Auslöser für Protest und Widerstand sind in der österreichischen Umweltbewegung von besonderer Bedeutung: Einerseits der Konflikt um das Atomkraftwerk in Zwentendorf im Tullnerfeld und andererseits die Verhinderung des Baus des Wasserkraftwerks in der Hainburger $\mathrm{Au}$, der in der Institutionalisierung des Nationalparks Donau-Auen resultierte. Der Widerstand gegen Zwentendorf wurde von einer heterogenen Protestbewegung getragen (Pesendorfer, 2007). Mit einer Volksabstimmung 1978 wurde letztlich mit einer knappen Mehrheit von 50,47 \% gegen die Inbetriebnahme gestimmt (Pelinka, 1985; Gottweis, 1997).

Den zweiten Höhepunkt des umweltpolitischen Engagements markiert die Besetzung der Hainburger Au im Dezember 1984 (Wösendorfer, 1988): Nach einem von der österreichischen Hochschüler/-innenschaft initiierten Marsch mit mehreren tausend Teilnehmer/-innen blieben einige in der Au, um dort Baumrodungen mit Mitteln zivilen Ungehorsams zu verhindern, was von der Polizei in umstrittener Weise beantwortet wurde. Massive Proteste dagegen veranlassten die Regierung schließlich, den sogenannten Weihnachtsfrieden auszurufen, woraufhin die Rodungen beendet wurden und der Nationalpark Donau-Auen entstand (Gottweis, 1997). Durch diese Protestbewegungen angestoßen, konnte Umweltschutz erfolgreich in der Politik verankert und Möglichkeiten der Bürger/-innenbeteiligung geschaffen werden, die Grüne Alternative entstand, welche erstmalig 1986 in den Nationalrat einzog (Dolezal, 2016). Es folgte die Ausdifferenzierung und teilweise Institutionalisierung der Umweltbewegung. Bis in die ersten Jahre des 21. Jahrhunderts wurde umweltpolitisches Engagement vor allem von Umweltschutzorganisationen, wie Global 2000, WWF Österreich oder Greenpeace getragen. Aktionsgruppen, wie System Change, not Climate Change! setzen sich nun zusätzlich seit einigen Jahren mit der Thematik auseinander (Dolezal \& Hutter, 2007; Krams \& Brand, 2019, S. 12f.).

Nach della Porta und Parks (2013) werden Umweltthemen verstärkt mit dem Terminus der Klimagerechtigkeit verbunden. Auch die FFF fordern (im Vergleich zu früheren Bewegungen) einen radikalen Wandel der (globalen) Klimapolitik, der nicht allein an der Umsetzung umweltpolitischer Maßnahmen ansetzt, sondern mehr die Idee nachhaltiger Entwicklung verfolgt und ebenso auf eine grundsätzliche Transformation der Wirtschaftsweise und Veränderung des Lebensstils zielt. Nach jahrzehntelangem institutionalisiertem umweltpolitischem Engagement kamen mit der FFF-Bewegung, die sich im Dezember 2018 in Wien formierte, wieder Streiks und Proteste in den öffentlichen Raum. FFF Austria versteht sich als Netzwerk von individuellen Akteur/-innen, vorwiegend von Jugendlichen und jungen Erwachsenen, wobei die große Zahl an jungen Frauen unter den Aktivist/-innen auffällt. Die überwiegende Mehrheit der Teilnehmer/-innen stammt aus der Mittelschicht (Daniel \& Deutschmann, 2020; Daniel in diesem Heft) - ähnlich wie bei den Protesten gegen das Atomkraftwerk Zwentendorf.

FFF mobilisierte im Jahr 2019 einerseits freitäglich zu den Schulstreiks, mit Bezug auf die kontinuierlichen Streiks 
Greta Thunbergs, andererseits organisiert(e) FFF im Rahmen zivilgesellschaftlicher Bündnisse die Global Earth Strikes. Diese Klimaproteste wurden in über 150 Ländern organisiert und unterstreichen damit auch die globale Dimension der Bewegung. Die FFF-Proteste eröffneten so eine neue Phase in der Geschichte der österreichischen Umwelt- und Klimabewegung. Zwar war die Umweltthematik seit Jahrzehnten bereits ein wichtiger Mobilisierungsauslöser in Österreich, doch gelang es erst den FFF-Protesten, das Thema Klimapolitik in die breite Öffentlichkeit zu tragen. Greta Thunbergs Streik verbunden mit einer Schulverweigerung inspirierte eine weltweite Welle des Engagements und die nachdrücklichen Forderungen der Schüler/-innen erreichten weltweit mediale Aufmerksamkeit. Die unmittelbar erfahrbar gewordene Problematik (z.B. durch Extremwetterereignisse) spielt dabei ebenfalls eine besondere Rolle, ebenso wie die Unterstützung der Wissenschaft, die für die Bewegung einen zentralen externen Legitimationsanker darstellt (Daniel \& Deutschmann, 2020). Die Schuld für ausbleibende Lösungen wird in diesem Kontext recht eindeutig der Politik zugewiesen (Daniel et al., 2020). Im Falle der FFF ging es somit vor allem um Wissensaneignung zu Fragen rund um die Klimakrise und ihrer Folgen, der Entwicklung von Lösungsvorschlägen ebenso wie die Organisation von Öffentlichkeitsarbeit (https://fridaysforfuture.at/ueber-uns; https://fridaysforfuture.at/forderungen) (s. auch Costa \& Wittmann in diesem Heft).

Junge Menschen, die sich bei FFF engagieren, formulieren z.B. in den Medien gut informierte Statements über die Bedeutung und die Wirkung des Klimawandels oder arbeiten an öffentlichkeitswirksamen (Protest-)Konzepten (wie Schuloder Klimastreiks). Engagement eröffnet so informelle Lernräume, die weitere Bildungsprozesse anregen und zudem formelle (Weiter-)Bildung bewirken können. Damit zeigt sich also nicht nur ein transformatives Potenzial auf der Ebene von Bewegungsstrukturen, sondern ebenso auf der Mikroebene der Akteur/-innen im Sinne transformativer Lern-und Bildungsprozesse, die auch im Rahmen einer Bildung für nachhaltige Entwicklung angestrebt werden.

\section{Soziale Bewegungen als transformative Lern- und Bildungsräume}

Am Beispiel der österreichischen Ökologie- und Umweltbewegung und anhand der neuen Proteste der FFF, lassen sich abschließend Verbindungen zwischen strukturellen Transformationsprozessen und subjektiven und organisatorischen Lern- und Bildungsprozessen aufzeigen. Auf der Ebene der Organisationen zeigten sich im Bereich der Umweltthematik zwei Institutionalisierungsprozesse: zum einen eine Etablierung der Grünen Partei, zum anderen die Herausbildung von Nichtregierungsorganisationen. Mit den FFF entwickelte sich eine neue (globale) Bewegung, die einerseits lokal verankert ist und ihre Forderungen auch an lokalen bzw. regionalen Projekten anbindet (vgl. https://fridaysforfuture.at/forderungen), andererseits eine globale Perspektive auf die Klimakrise einnimmt und der Politik die Einhaltung politischer Abkommen - vor allem der Pariser Klimakonvention - abverlangt.

Innerhalb der Bewegung organisieren sich junge Menschen, lernen dabei selbst über den Klimawandel und seine
Folgen, setzten sich mit wissenschaftlichen Befunden aus der Klimaforschung und politischen Wirkformaten auseinander. Sie fordern politisches und generationsübergreifendes individuelles Handeln ein (ohne einen Generationenkonflikt zu forcieren, vgl. dazu Daniel \& Deutschmann, 2020).

Die erarbeiteten Vorschläge und Entwürfe von politischer Gestaltung setzen an jenen anderer Akteur/-innen in der Klima(gerechtigkeits-)bewegung an, streben nach lokaler Verankerung der weltweiten Bewegung und versuchen zugleich die Probleme als globale Probleme zu verstehen. Soziale Bewegungen bieten so einen nach innen und nach außen gerichteten transformativen Lern-Bildungsraum an. Was Truemann (2013) für Büger/-inneninitiativen beschreibt, kann auch für die FFF-Bewegung festgestellt werden: Sie schaffen einen Lern-Handlungsraum, der auch als Türöffner für politische Partizipation und Bildung fungiert. In besonderer Weise trifft das auf die zu einem großen Teil noch nicht wahlberechtigten Aktivist/-innen zu, für die das Engagement in besonderer Weise erstes politisches Handeln ermöglicht (de Moor et al., 2020). Zudem haben die FFF, jenseits der bereits etablierten politischen Parteien und NGOs bewirkt, dass Umweltthemen und die Klimakrise globale Aufmerksamkeit erhalten, über generationale und strukturelle Grenzen hinweg. So werden vertiefende Diskurse um (lokale) Maßnahmen und um (globale) Klimagerechtigkeit angeregt.

Im Sinne bildungspolitischer Aufmerksamkeit sollten neben Themen des Bildungsauftrags und der Schulpflicht im Zusammenhang mit den Schulstreiks über die Funktion und Bedeutung von Bildungsinstitutionen und gesellschaftlichen wie individuellen Lern-und Bildungsprozessen (in) sozialen Bewegungen reflektiert werden; gerade auch vor dem Hintergrund der Forderungen nach einem Lernen für einen nachhaltigen und gerechten Wandel.

\section{Anmerkungen}

1 Gaigg, V. et al. (2021): Die Köpfe hinter den „Querdenker“-Demos. Zugriff am 15.01.2021 https://www.derstandard.at/story/2000123339446/die-koepfe-hinter -den-querdenker-demos

2 An anderer Stelle habe ich herausgearbeitet, wie studentische Bewegungen in Mali und Kenia, die sich im Zuge von Demokratisierungsprozessen als studentische Interessenvertretungen institutionalisierten, nunmehr anstelle bzw. zusätzlich zu politischen Agenden (wie der Demokratisierung) von sozialen Aufstiegsaspirationen der Repräsentat/-innen und potenziellen (politischen) Karrieren geprägt sind. Diese Prozesse habe ich rekonstruiert und gezeigt, dass sich über mehrere Generationen die Aspirationen studentischer Aktivist/-innen bzw. Repräsentat/-innen (in institutionalisierten) Strukturen verändert haben und dabei in einem interdependenten Prozess mit der Rekrutierung und Legitimierung (politischer) Eliten stehen (Deutschmann, 2020).

3 Diese Darstellung lehnt sich an eine ausführlichere Darstellung der Ereignisse an anderer Stelle an (vgl. hierzu Daniel et al., 2020). 


\section{Literatur}

Ahlemeyer, H. W. (1989). Was ist eine soziale Bewegung? Zur Distinktion und Einheit eines sozialen Phänomens. Zeitschrift für Soziologie, 18, 175-191. https:// doi.org/10.1515/zfsoz-1989-0301

Amenta, E., Caren, N., Fetner, T., \& Young, M. P. (2002). Challengers and states: Toward a political sociology of social movements. Research in Political Sociology, 10, 47-83. https://doi.org/10.1016/S0895-9935(02)80018-3

Daniel, A. \& Deutschmann, A. (2020). Umweltbewegung revisited? Fridays for Future in Wien: Profil und Einstellungen einer neuen Protestbewegung. Working Paper No. 9. Daniel, A., Deutschmann, A., Buzogány, A. \& Scherhaufer, P. (2020). Die Klimakrise deuten und Veränderungeneinfordern: Eine Framing-Analyse der Fridays for Future. SWS-Rundschau, 60(4), 365-384.

della Porta, D. \& Parks, L. (2013) Framing-Prozesse in der Klimabewegung: VomKlimawandel zur Klimagerechtigkeit. In M. Dietz H. Garrelts (Hrsg.) Die internationale Klimabewegung: Ein Handbuch (S. 39-56). Wiesbaden: Springer.

de Moor, J. et al. (Hrsg.). (2020). Protest for a Future II: Composition, Mobilization and Motives of the Participants in Fridays for Future Climate Protests on 20.-27. September 2019 at 19 Cities Around the World. Zugriff am 29.06.2020 https:// tinyurl.com/y42r5bm9

Deutschmann, A. (2020): Studierende als Akteure im politischen Wandel Afrikas. Ein Vergleich studentischer Mobilisierung und Organisation in Mali und Kenia 1960-2018. Wiesbaden: Springer. https://doi.org/10.1007/978-3-658-29878-4

Dolezal, M. (2016). The Greens in Austria and Switzerland. In E. Van Haute (Hrsg.), Green Parties in Europe (S. 15-42). Abingdon: Routledge.

Dolezal, M. (2019). Wogegen Österreich aufbegehrt. Zugriff am 08.09.2021 https:// tinyurl.com/yxq4xgnb.

Dolezal, M. \& Hutter, S. (2007). Konsensdemokratie unter Druck. Politischer Protest in Österreich 1975-2005. Österreichische Zeitschrift für Politikwissenschaft, 3, 337-352.

Ebbinghaus, A. (2008). Die 68er. Schlüsseltexte der globalen Revolte. Wien: Promedia. Gottweis, H. (1997). Neue Soziale Bewegungen in Österreich. In H. Dachs et al. (Hrsg.) Handbuch des politischen Systems Österreich. Die Zweite Republik (S. 359-368). Wien: MANZ.

Gottweis, H. (2000). Politische Mobilisierung. BürgerInnenbewegung und Ansätze zur Ausbildung neuer Organisationsformen von Politik in Österreich. In Forum Politische Bildung (Hrsg.) Zum politischen System Österreich. Zwischen Modernisierung und Konservativismus. (S. 60-67). Wien u. Innsbruck: Studien.

Haselwanter, M. (2020). Die Unterbrechung des Zeitregimes als Möglichkeit auf Bildung. Fridays for Future - mehr als ein (Schul-)Streik. Magazin erwachsenenbildung.at. Das Fachmedium für Forschung, Praxis und Diskurs, 41.

Heuwieser, M. (2016). Geld wächst nicht auf den Bäumen - oder doch? Warum die Natur und deren "Leistungen" $z u$ Waren gemacht werden. Berlin: FDCL.

Krams, M. \& Brand, U. (2019). Die Klimakrise: Ursachen, Handlungsansätze und Kritik. In Informationen zur Politischen Bildung, Umwelt-Klima-Politik, 45, 2019, 5-14.

McAdam, D., McCarthy, J. D., \& Zald, M. N. (1996). Comparative perspectives on social movements : political opportunities, mobilizing structures, and cultural framings. Cambridge: Cambridge University Press. https://doi.org/10.1017/CBO97805118 03987

McCarthy, J. \& Zald, M. (1977). Resource Mobilization and Social Movements: A Partial Theory. The American Journal of Sociology, 82(6), 149-172. https://doi. org/10.1086/226464

Miethe, I. (2017). Soziale Bewegungen, Bildungsaufstieg und Bildungsreform: fünf Verbindungen von Bildung und Bewegung. DIE Zeitschrift für Erwachsenenbildung, 2, 36-38.

Miethe, I. \& Roth, S. (2016). Bildung und soziale Bewegungen - eine konzeptionelle Einführung. Forschungsjournal Soziale Bewegungen, 29(4), 20-29. https://doi. org/10.1515/fjsb-2016-0262

Offe, C. (1990). Reflections on the Institutional Self-Transformation of Movement Politics: A Tentative Stage Model. In R. J. Dalton \& M. Kuechler (Hrsg.), Challenging the political order: new social and political movements in Western democracies. (S. 323-350). Cambridge: Polity Press.

Overwien, B. (2013). Informelles Lernen in politischer Aktion und in sozialen Bewegungen. In Außerschulische Bildung. Zeitschrift des Arbeitskreises außerschulischer Bildungsstätten, 3(2013), 247-255.

Overwien, B. (2016). Informelles Lernen und politische Bildung. In M. Rohs (Hrsg.), Handbuch informelles Lernen (S. 399-412.). Wiesbaden: Springer VS. https://doi.org/10.1007/978-3-658-05953-8_38

Pelinka, A. (1985). Hainburg - mehr als nur ein Kraftwerk. In A. Khol et al. (Hrsg.), Österreichisches Jahrbuch für Politik 1985 (S. 93-109). Wien: MANZ.

Pelinka, A. (1991). The Study of Social Movements in Austria. In D. Rucht (Hrsg.), Research on Social Movements (S. 230-247). Frankfurt a. M.: Boulder.
Pelinka, A. (1998). Austrian Political Culture: From Subject to Participant Orientation. In K. R. Luther \& P. Pulzer (Hrsg.), Austria 1945-95 (S. 109-119). Aldershot et al.: Routledge. https://doi.org/10.4324/9780429463297-6

Pesendorfer, D. (2007). Paradigmenwechsel in der Umweltpolitik: von den Anfängen der Umwelt- zu einer Nachhaltigkeitspolitik: Modellfall Österreich? Wiesbaden: Springer.

Rodemann, S. (2009). Gestaltungskompetenz durch freiwilliges Engagement bei Greenpeace. In M. Brodowski, U. Devers-Kanoglu, B. Overwien, M. Rohs, S. Salinger \& M. Walser (Hrsg.), Informelles Lernen und Bildung für eine nachhaltige Entwicklung Beiträge aus Theorie und Praxis (S. 103-112). Leverkusen u. Opladen: Barbara Budrich. https://doi.org/10.2307/j.ctvddzjqt.13

Roth, R. (1991). Gegen Eliten oder Gegeneliten? Grüne und neue soziale Bewegungen in der politischen Kultur der Bundesrepublik. In: S. R. Klingemann, B. Weßels (Hrsg.), Politische Klasse und politische Institutionen (S. 434-465). Wiesbaden: VS Verlag für Sozialwissenschaften. https://doi.org/10.1007/978-3-32294153-4_18

Rucht, D. (1994). Modernisierung und neue soziale Bewegungen. Frankfurt a. M.: Campus.

Rucht, D., Koopmans, R., \& Neidhardt, F. (1998). Acts of dissent. New Developments in the Study of Protest. Berlin: edition sigma. https://doi.org/10.1007/978-3322-97316-0_87

Singer-Brodowski, M. (2016). Transformative Bildung durch transformatives Lernen. Zur Notwendigkeit der erziehungswissenschaftlichen Fundierung einer neuen Idee. Zeitschrift für internationale Bildungsforschung und Entwicklungspädagogik, 39(1), 13-17.

Tentative Stage Model. In R. J. Dalton \& M. Kuechler (Hrsg.), Challenging the political order: new social and political movements in Western democracies. (S. 323350). Cambridge: Polity Press.

Trumann, J. (2013): Lernen in Bewegung(en): Politische Partizipation und Bildung in Bürgerinitiativen, Edition Politik: Transcript. https://doi.org/10.14361/transcript.97 83839422670

Wösendorfer, H. (1988). »Hainburg «- Krise, Konflikt und Planung. In R. Burger, E. Matzner, \& A. Pelinka (Hrsg.), Verarbeitungsmechanismen der Krise (S. 113-145). Wien: Wilhelm Braumüller.

\section{Dr. Anna Deutschmann}

ist Sozialwissenschaftlerin. Ihre Interessen liegen u.a. im Bereich der politischen Transformationen, sozialer Bewegungen, sozialer Ungleichheit und Bildungssoziologie. Sie arbeitet in einer NGO sowie als Lektorin an der Universität Wien. 